\title{
Cancer labs reach beyond exhausted T cells
}

\author{
T cells race to the scene in cancer and infection. To tease out what is special in cancer, scientists widen their scope \\ and tool sets.
}

\section{Vivien Marx}

/ / | ' 'm a basic scientist. I did not get into these studies to try to cure cancer,

I got into them because I wanted to know how T cells worked," immunologist Jim Allison said at a news conference when he received the Nobel Prize in Physiology or Medicine. When he realized cell-intrinsic molecules can act as negative regulators of the immune response, he wondered how this could be leveraged to unleash T cells for cancer treatment. This led to checkpoint blockade, in which immune responses are enhanced by removing certain types of T cell 'brakes' or checkpoints.

Cancer cells can evade the immune system by diminishing the T cell's ability to kill, says University of Chicago researcher Melody Swartz. By stimulating checkpoint pathways such as cytotoxic T-lymphocyteassociated antigen 4 (CTLA-4) and programmed cell death protein 1 (PD-1) on T cells, cancer exploits these pathways to its own advantage.

Allison's collaborator Padmanee Sharma, an investigator, immunotherapist and his wife, pointed out in an interview that checkpoint blockade has been successful for treating several different cancer types. But in many instances the treatment does not work well or resistance develops, for unclear reasons. "So we're going back to basic lab research," she noted.

The immune system detects that a tumor's antigens are altered proteins, says Swartz. Usually these are mutated proteins or proteins typically turned off after a certain developmental phase, but they are overexpressed in a tumor. "T cells can recognize these antigens and kill tumor cells," she says. T cells can leave the bloodstream and enter a solid tumor as tumor-infiltrating lymphocytes (TILs). But in their cancer battle, they can become exhausted.

\section{Exhaustion happens}

The term $\mathrm{T}$ cell exhaustion originated in the description of mice unable to clear a chronic infection. Exhausted T cells tend to produce fewer cytokines and have higher expression of inhibitory receptors such as PD-1 or CTLA-4. Exhaustion likely evolved as the immune system's mode of

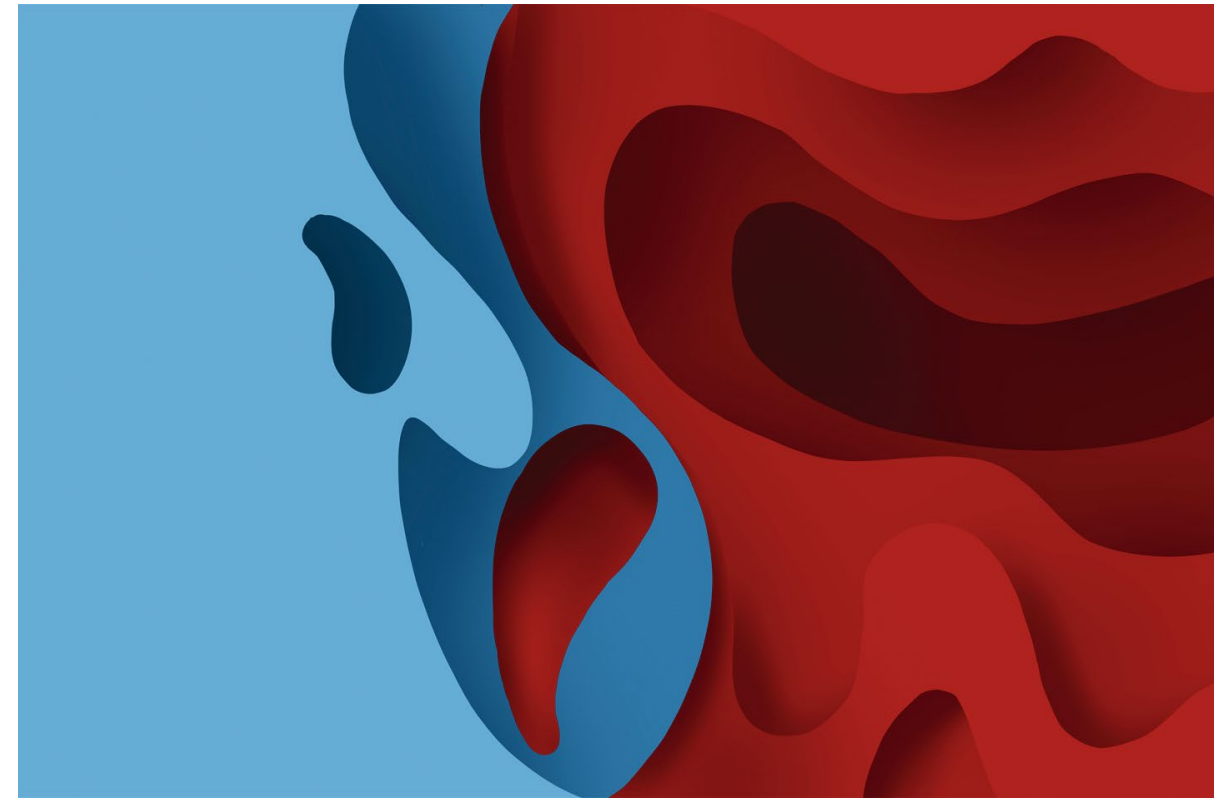

Tumors are sometimes classified as 'hot' or 'cold'. A hot, inflamed tumor lets T cells in where they can kill cancer cells. Scientists are trying to figure out how to maintain T cell striking power in hot tumors and get $\mathrm{T}$ cells in the door in cold tumors. Credit: mikroman6/Moment/Getty

chastening autoimmune reactions. In that sense, notes John Wherry of the University of Pennsylvania, "exhausted T cells are not inherently good or bad" ${ }^{1}$. But in cancer, exhausted T cells cannot attack cancer cells effectively. Scientists recently discovered that a transcription factor called thymocyte selection-associated HMG-box protein (TOX) is an important choreographer of T cell exhaustion ${ }^{2-6}$.

T cell exhaustion occurs in infection and cancer, says Vanderbilt University Medical Center researcher Mary Philip, who co-led the TOX work ${ }^{4}$. In chronic viral infection, $\mathrm{T}$ cells start out functional and then, as the infection keeps stimulating them, become exhausted and lose function, "though they can retain enough function to achieve a stalemate with the virus," she says. "In cancers, T cells often enter straight into a 'non-functional' state.'

Even when they are in that state, says Andrea Schietinger, a researcher at Memorial Sloan Kettering Cancer Center, who co-led this work, the tumor continues to stimulate T cells, which turns on TOX and the cells look exhausted, she says. "These dysfunctional T cells in tumors generally don't do anything," she says. "They just hang around and let the tumor grow." In their research, they looked at TOX in CD8+ TILs and peripheral blood mononuclear cells from people with melanoma, breast cancer, lung cancer or ovarian cancer, as well as assessing the effect of deleting Tox in vitro and in mice. They performed

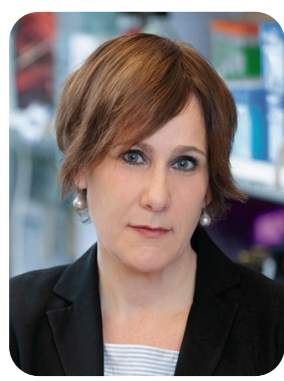

One next big question calling for an answer: what controls whether $T$ cells are functional or not, says Andrea Schietinger, a researcher at Memorial Sloan Kettering Cancer Center. 


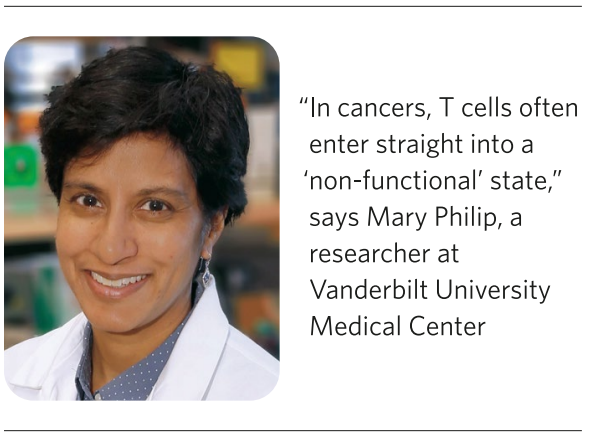

RNA-sequencing and used Assay for Transposase-Accessible Chromatin Using Sequencing (ATAC-seq), a method for characterizing how tightly or loosely DNA is packaged across the whole genome.

Functional and dysfunctional $\mathrm{T}$ cells have identical DNA sequence; it's their epigenetics, how those sequences are expressed and regulated, that determine the cell's fate and function, says Philip. An advantage of ATAC-seq, she says, is that it can be used with low cell numbers, a typical constraint when dealing with $\mathrm{T}$ cells from mouse and human tumors.

Unlike T cells in an acute infection, $\mathrm{T}$ cells in tumors become exhausted and lose their ability to kill, says Schietinger. As they scoured transcription factors uniquely expressed in T cells, TOX topped the list, she says. They first found TOX in tumor mouse models and then found it expressed at high levels in T cells in all human tumors they studied - in breast and lung tumors, in melanoma and in ovarian cancer; "and that's when we were convinced that we were on to something," she says.

It was a surprise, says Philip, that TOX regulates many features of $\mathrm{T}$ cell exhaustion but does not control $\mathrm{T}$ cell effector function, which occurs when an antigen activates a naïve $\mathrm{T}$ cell to an armed and ready $\mathrm{T}$ effector cell. Tumor-infiltrating $\mathrm{T}$ cells with a knocked-out Tox gene no longer looked exhausted, but remained dysfunctional and did not kill tumor cells, says Philip. Schietinger says, "I think this is the next big question that needs to get answered: what controls whether $\mathrm{T}$ cells are functional or not."

Exhausted T cells have a "unique epigenetic landscape," as Wherry has phrased it. He and colleagues have investigated that landscape with immunoprecipitation followed by mass spectrometry. They looked at proteins bound to TOX that induce epigenetic changes. Among the proteins are ones involved in chromatin organization and remodeling, RNA processing, and translation. TOX, the authors note, is "the key inducer of canonical features of exhaustion." They note that exhausted T cells are a "distinct cell type," separate from $\mathrm{T}$ cell relatives such as T effector cells or T memory cells.

\section{Thinking CAR-T}

T cell exhaustion shapes research related to engineering T cells to attack tumors, such as chimeric antigen receptor (CAR) T-cell approaches ${ }^{7}$. Carl June at the University of Pennsylvania and colleagues advanced CAR-T therapy, in which a patient's T cells are equipped with synthetic receptor molecules and then infused into the patient. As Stanford University researchers Crystal Mackall, Howard Chang and their colleagues point out, CAR-T therapy is successful in a "small subset of patients".

But T cell exhaustion is an issue. Chang and colleagues applied single-cell ATAC-seq (scATAC-seq) to identify tens of thousands of 'accessible' chromatin regions and locate genes associated with exhaustion?. "A dominant signal in our scATAC-seq work suggested involvement of the c-JUN AP1 pathway," says Chang. Together with Crystal Mackall's group, they engineered mice to overexpress c-JUN, an AP-1-family transcription factor. The CAR-T cells became resistant to exhaustion and were functionally much more potent in their attacks on cancer, he says. They tested the cells in immunocompromised mice that had been inoculated with leukemia or osteosarcoma.

Several key transcription factors appear to drive T cell exhaustion, says Chang, in whose lab ATAC-seq was developed. In ATAC-seq, nuclei are suspended with transposase, followed by barcoding and sequencing. He and his team also developed scATAC-seq by integrating the method into the programmable Fluidigm microfluidic platform. The first version of scATAC-seq could handle 96 individual cells per assay. "We knew that we had to scale the assay up to handle thousands if not tens of thousands of cells to tackle highly heterogeneous cells that are present in complex tissues," says Chang. Droplet-based scATAC-seq gave

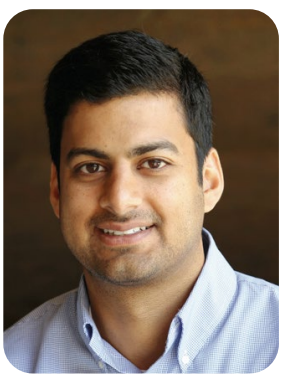

Exhausted T cells have a chromatin pattern that is "just as distinct as any other commonly considered T cell state," says Ansuman Satpathy, Stanford University School of Medicine. Credit: N. Cyr, Stanford University them massively parallel scale for assessing the genome and its DNA-binding proteins. In one experiment, they analyzed 200,000 single cells. There are various protocols for scATAC-seq, and 10X Genomics, for one, offers an instrument.

Chang is optimistic that single-cell epigenomics will help improve cancer immunotherapy. Wherry and others have reported that exhausted $\mathrm{T}$ cells have a distinct epigenetic state and chromatin pattern, so "T cell exhaustion was potentially a good system to apply our new scATAC-seq technology." Chang and colleagues looked at intratumoral $\mathrm{T}$ cell exhaustion with patient samples and used scATAC-seq's scale to tease out exhausted $\mathrm{T}$ cells for analysis.

Separately, they combined sequencing of TCRs and single-cell RNA-sequencing to assess samples before and after PD-1 blockade. Exhausted T cells appear to not be reinvigorated after PD-1 blockade. Rather, says Chang, a new group of T cells enters the tumor and expands. "This 'clonal replacement' concept is an important new direction for cancer immunotherapy," he says.

Exhausted T cells have a chromatin pattern that is "just as distinct as any other commonly considered T cell state," says Ansuman Satpathy, also at Stanford and coauthor of the work. "Using the singlecell data, we could map the developmental trajectory from $\mathrm{T}$ cell activation to exhaustion and nominate regulatory elements and transcription factors that may be critical for this process."

Another valuable technique for studying T cell exhaustion, says Schietinger, is spatial transcriptomics, given that $\mathrm{T}$ cell properties will vary in different bodily locations and even within a tumor. "We are excited by the new technologies emerging to allow us to combine transcriptomics with location information," she says. "TOX got a lot of buzz," she says. Among the next research tasks is "how we make lousy T cells into cancer killer T cells, and this has likely nothing to do with TOX," she says. "So stay tuned."

\section{CAR and gene-editing}

CAR-T therapies are approved to treat some hematological cancers. Scientists are exploring ways to avoid cytokine release syndrome (CRS), a possible grave side effect of CAR-T therapy, and are looking into how CAR-T cells might be used in solid tumors. That's when it's key for T cells to avoid exhaustion and persist in the tumor microenvironment.

Chang, together with Carl June's group, has gene-edited T cells using CRISPR - in particular, the genes encoding the 
TCR $\alpha$ and $\beta$ chains ${ }^{10}$. "Our single-cell analysis actually suggests that gene editing of PD-1 did not have a major effect on T cell persistence," says Chang. What may help with T cell persistence, they found, is knocking out endogenous $\mathrm{T}$ cell receptor genes. One issue with CARs is mispairing of engineered TCRs with endogenous ones. The mispairing risk is lower when endogenous TCRs are out of the way, he says. This interpretation also agrees with findings about the advantages of inserting cancer-specific TCRs into the endogenous TCR locus.

CRISPR editing of $\mathrm{T}$ cells need not be limited to genes, says Satpathy, coauthor of the gene-editing work. "Editing cell-contextspecific enhancers could also be beneficial to achieve more precise control of gene expression," he says.

One challenge with currently approved CAR T approaches, says Swartz, is that CAR T cells are designed to identify one particular tumor antigen, such as CD19 in the case of lymphoma. "Tumor cells evolve and mutate, and can downregulate such antigens," she says. Tumors can develop mechanisms to inactivate these $\mathrm{T}$ cells. "Newer approaches target multiple antigens, but I think the bigger problem is that we need approaches that target a broader range of tumor antigens and that are longerlasting," she says. This will take more basic research about, for example, the subtleties of bystander $\mathrm{T}$ cell activation, antigen spreading, and what it takes to maintain a robust pool of memory $\mathrm{T}$ cells, she says.

\section{It's the environment}

Cancer cells express major

histocompatibility class I (MHC I) proteins on their surface, which patrolling T cells pick up on, says Philip. T cells have varying specificities for different peptides, and when a particular $\mathrm{T}$ cell recognizes peptides on a cancer cell's MHC I, that T cell is activated. "But the context, or microenvironment, plays a big role in how the $\mathrm{T}$ cell will function once it is activated," she says. In cancer, the microenvironment can lead the $\mathrm{T}$ cell to be non-functional and unable to kill cancer cells. As cancer cells accumulate, they will continue to stimulate the $\mathrm{T}$ cell through their peptide-MHC I, and T cells will become exhausted.

The scale of the scATAC-seq method proved helpful for analyzing issues related to the tumor microenvironment, says Satpathy. In a single assay, they could capture profiles of all the cells in the tumor microenvironment. For example, they saw an 'exhaustion-like' differentiation trajectory in $\mathrm{CD}^{+}$cells that led to a type of $\mathrm{T}$ cell called a follicular helper $\mathrm{T}$ cell. These cells

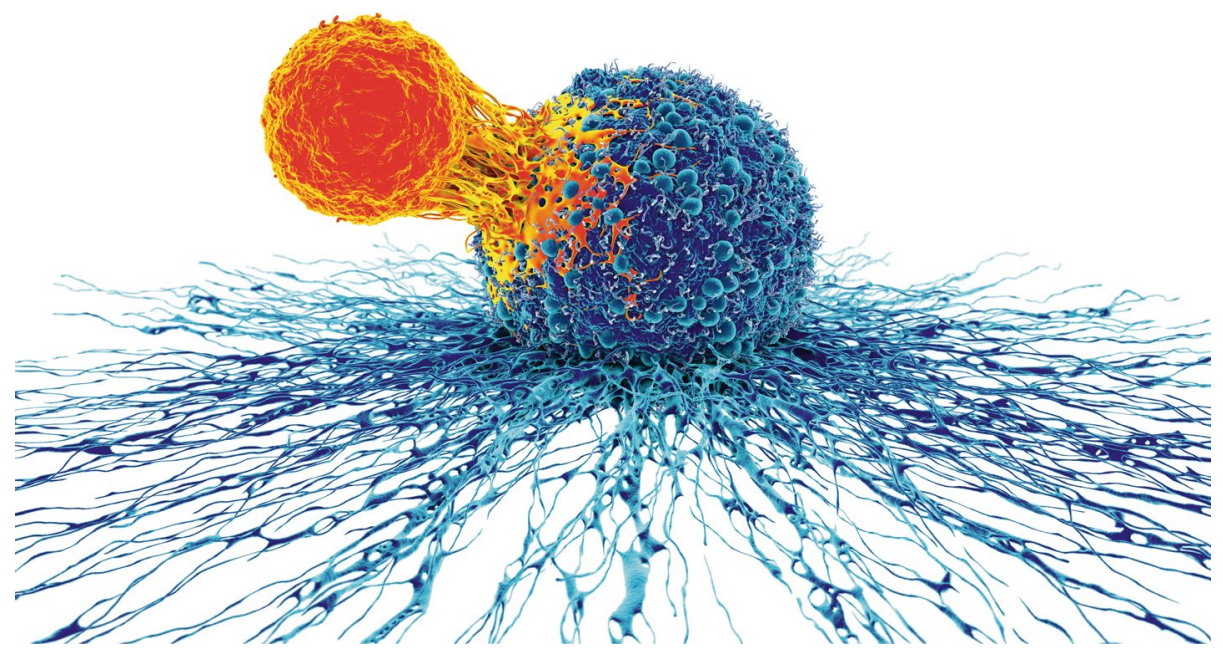

T cells (orange) can attack and kill cancer cells (blue). But in this battle, seen here in an artist's rendering, T cells can become exhausted. Researchers seek ways to reinvigorate them. Credit: R. Harris/ Science photo library/Getty

are particularly good at supporting $B$ cell responses, he says. In patients whose disease responded to immunotherapy, the team saw an expansion of $\mathrm{CD}^{+}$exhausted $\mathrm{T}$ cells. CD4 ${ }^{+} \mathrm{T}$ follicular helper cells and $B$ cells were present, too, all of which suggests a coordinated immune response. Other groups have shown that the presence of $B$ cells in the tumor microenvironment "is a positive indicator of checkpoint response," he says.

The tumor microenvironment appears to be immunosuppressive in many ways. The immune response is shaped by so many different factors, and immunotherapy outcomes integrate all of these effects, says Swartz. There are codependencies in this interaction between tumor and microenvironment, too. In her lab and others, researchers explore the system dynamics - how the different cell types, inhibitory and stimulatory receptors, and cytokines all work together and influence each other.

Transcriptomics does not yield insight about interactions, just the gene expression state of each cell. "We actually need better tools to understand the complex dynamics of immune responses," says Swartz. She and her team are working on an in vitro model of the tumor immune microenvironment that uses ex vivo tumors and tumor-draining lymph nodes. "This still needs further development to be useful, but I think such tools will be critical for understanding the way the cells and the microenvironment coevolve and interact during an immune challenge." Beyond T cells and the microenvironment, some groups exploring the modification of natural killer (NK) cells to express an anti-CD19 CAR.

\section{Models}

"It's always great when a model works," says Janell Richardson, a field applications scientist at Taconic Biosciences, which develops rodent models, including genetically engineered ones, for customers. "But, most importantly, we also want to know, when it doesn't work, why didn't it work." When creating mouse models for cancer researchers, especially in immunooncology, she and her team want to understand the predictive validity of models.

It has become clear that a tumor's total mutational burden matters, not just that the tumor, for example, expresses high levels of PD-L1. Much is known about 'hot' tumors, with inflammation levels that indicate many TILs. "I think the difficult part is: what about a cold tumor?" she says. Cold tumors are sometimes called "deserts" because of their lack of immune cells. The research question "is there a way to transition a cold tumor to a hot tumor?" is one of many open ones, she says.

Among her customers, she sees basicresearch-oriented moves. One example is gazing beyond a focus on a single target such as PD-1. Those approaches worked well in some cancers, "but we see a lot of frustration," she says, "because certainly, it's not successful across the board." Going back to basics involves more complex, broaderscope questions. "Now we understand that it's also not just about the T cell," she says. She sees labs exploring the roles of stromal cells in the tumor microenvironment, myeloid-derived suppressor cells, 


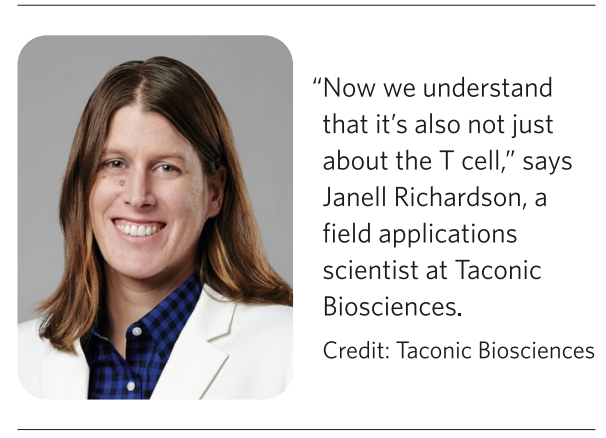

tumor-associated macrophages, inhibitory receptors and cytokines. There are supporting lymphoid and myeloid cells and there is cross-talk between them, all of which helps to explain the immune system response to cancer. Tumor-associated macrophages can, it seems, directly influence the overall immunosuppressive environment, as can myeloid-derived suppressor cells. It will be fruitful to understand the mechanisms underlying $T$ cell exhaustion. "If they matter in exhaustion, then they're going to matter in reinvigoration," says Richardson.

An in vivo system is well suited to exploring the complex, multiparallel system of internal and externally based signals, she says. But mice have their limitations. "Some mice do not show cytokine release syndrome," she says. One immunotherapy clinical trial led to severe, life-threatening cytokine storms. A much higher dose had been tested on non-human primates that hadn't shown CRS. "It tells you the complexity and the species specificity of this system," she says.

CRS has been shown in humanized immune system models such as such as the $\mathrm{CD} 4^{+}$engrafted immunodeficient NOD/ SCID $\gamma$ mouse. For it to occur, the lymphoid and myeloid immune lineages need to be present, says Richardson. But these mice "typically tend to be a very lymphoiddominant lineage model," she says. "The models are far from perfect," she says. Better ways to model CRS are needed so labs can learn what underpins this reaction.

Richardson and her team are advancing humanized immune system models to gain a deeper understanding of engraftment. In engraftment, peripheral blood mononuclear cells or fetal cord blood-derived hematopoietic stem cells are engrafted into 'super-immunodeficient' animals, which gives a lymphoid and, potentially, a myeloid compartment. Because these mice have an incomplete immune system, one with both human and mouse components, she reminds her customers to exercise caution when interpreting results.

She and her team are working on 'nextgen humanized immune system models': models with endogenous human cytokines. These models will contain cells typically absent or present at only very low levels in the super-immunodeficient mice and make it possible to ask more intricate research questions, she says.

Richardson advises customers to remember how results can differ between a syngeneic mouse model, which is "basically a mouse tumor in a mouse host" - one with the same genetic background and a humanized mouse model. Some immunotherapy studies have shown divergent results in the two model classes.

In applied R\&D, she observes some bridge-building. Traditionally, scientists worked in oncology or autoimmune disorders: "they would go about their work in siloed activities," she says. But scientists are now seeing that a target that has failed in immuno-oncology and did not "unhinge" inhibition might have promise for autoimmune disorders, where it's an explicit goal to inhibit the immune reaction.

\section{Hot or cold}

A hot tumor readily admits $\mathrm{T}$ cells, but once in, they can get trapped and repeatedly stimulated and become exhausted, says Schietinger. "A cold tumor does not open its doors to let T cells in." Hot and cold tumors call for different approaches: "in a hot tumor I need to figure out how to keep $\mathrm{T}$ cells from getting exhausted, and in cold tumors I need to figure out how I get my $\mathrm{T}$ cells in the door of the tumor."

Tumors that are more inflamed or more infiltrated with $\mathrm{T}$ cells generally respond better to checkpoint blockade, says Swartz. Some melanomas are lymphangiogenic and more prone to metastasize, and she and her team have found that lymphangiogenic tumors are more responsive to immunotherapy ${ }^{11}$. VEGF-C, a lymphangiogenic growth factor, upregulates the lymphoid homing chemokine CCL21. This attracts naive and memory $\mathrm{T}$ cells into the tumor, as well as dendritic cells, all which have the CCL21 receptor CCR7. "Thus, once an immunotherapy is initiated and $\mathrm{T}$ cell killing of tumor cells occurs, these 'bystander' naive and memory T cells can also be activated, which amplifies and broadens the immune response," she says. Inhibiting VEGF-C worsens the response to immunotherapy; "this was our surprising discovery - it shows that even while VEGF-C is considered bad in terms of metastasis, it can be good for immunotherapy."

Patients who respond to immunotherapy more frequently have more inflamed 'hot' tumors. This is relevant for checkpoint blockade "because if you don't have T cells there to begin with, it is not much use to prevent their exhaustion," she says. On the other hand, there are many other types of immunotherapy, such as CAR-T cells, dendritic cell vaccines, and protein or peptide vaccines. Pre-existing inflammation may play different roles in CAR-T cell approaches and checkpoint blockade.

Different tumors can have different barriers to immunotherapy, which are also different depending on the immunotherapy in question, says Swartz. Cells that also need to be considered include myeloidderived suppressor cells, M2 macrophages and regulatory $\mathrm{T}$ cells, all which inhibit cytotoxic $\mathrm{T}$ cell function. Cancer-associated fibroblasts can exclude T cell entry into the tumor, and there is expression of inhibitory molecules like PD-L1. In her view, scientists will want to characterize the overall immune microenvironment and strive for more detail, reaching beyond a classification of hot versus cold.

\section{Vivien Marx \\ Technology editor for Nature Methods. \\ e-mail:v.marx@us.nature.com}

Published online: 18 March 2020

https://doi.org/10.1038/s41592-020-0800-5

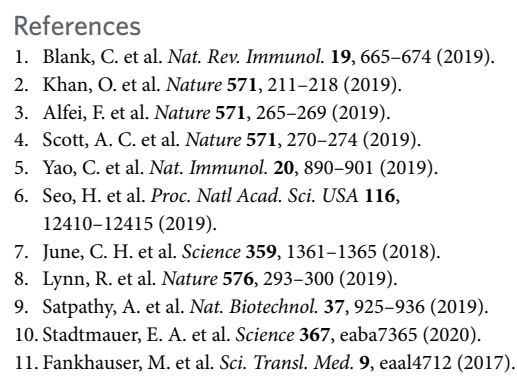

References

1. Blank, C. et al. Nat. Rev. Imm

2. Khan, O. et al. Nature 571, 211-218 (2019).

4. Scott, A. C. et al. Nature 571, 270-274 (2019).

5. Yao, C. et al. Nat. Immunol. 20, 890-901 (2019)

6. Seo, H. et al. Proc. Natl Acad. Sci. USA 116,

12410-12415 (2019)

8. Lynn, R. et al. Nature 576, 293-300 (2019).

9. Satpathy, A. et al Nat. Biotechnol, 37, 925-936 (2019).

11. Fankhauser, M. et al. Sci. Transl. Med. 9, eaal4712 (2017). 